\title{
An exact solution for tube and slit flow of a FENE-P fluid
}

\author{
P. J. Oliveira, Covilhã, Portugal \\ (Received September 20, 2001; revised March 11, 2002)
}

\begin{abstract}
Summary. A fully analytical solution is derived for rectilinear flow of a nonlinear viscoelastic fluid obeying the constitutive FENE-P model, under fully developed conditions. Both the plane case (slit flow) and the axisymmetric case (tube flow) are considered. Physical interpretation of the results is provided. The normal stress profile is found to vary in a non-monotone way with the dimensionless parameter characterizing viscoelasticity, the Deborah number $(D e)$. For Deborah numbers below a critical value (dependent on the extensibility parameter of the model $L^{2}$ ) the normal stress raises with elasticity, but this trend is reversed for values above the critical one. This effect is due to the competing influence of elasticity and shear thinning. Also, as a consequence of shear thinning the velocity profile becomes flatter as $D e$ increases and $L^{2}$ decreases, leading to higher flow rates for the same pressure drop.
\end{abstract}

\section{Introduction}

Interest in analytical studies of flow problems involving viscoelastic fluids has been growing, as a cursory overview of the contents of a specialized journal reveals; for example, Hayat et al. have tackled some time-dependent problems with the second-order Rivlin fluid [1], [2], and also with the Oldroyd-B fluid [3]. While these are useful theoretical works, it should be pointed out that the second-order fluid is hardly ever used to represent real fluids as it possesses a negative first-normal stress coefficient, contrary to what is observed, and the Oldroyd-B is a quasi-linear model having a constant viscosity. Models used to represent actual polymeric fluids are usually more complex than the above, being nonlinear in the stresses and with implicit differential constitutive equations relating stress with strain rates. Typical models of this kind, often used in simulation works, are the Phan-Thien - Tanner model [4], the FENE-P model [5] and the Giesekus model [6].

In this note we derive analytically the solution for the problem of fully developed flow of a FENE-P fluid flowing either along a circular cross-section tube or within the space between two parallel plates (slit flow). Due to the intrinsic nonlinearity of the FENE-P constitutive model, it has apparently gone un-noticed that such solutions can be easily worked out for the particular situation considered here, of fully-developed flow conditions, as will be shown. Analytical solutions for simple flows of viscoelastic fluids are useful per se, as they allow a complete description of the flow with given explicit expressions (in some cases the solution is only implicit), and are also useful to be applied as boundary conditions in computational simulations, with the benefit of allowing a reduction of the size of the computational domain.

Without the worry of being exhaustive, we give below a brief overview of some related analytical works. In the early days of rheology, Rivlin [7] gave the solution for Poiseuille 
flows of the Reiner-Rivlin fluid and, a few years later, Oldroyd [8] indicated how the solution for rectilinear flows of his general 8-constant fluid model could be constructed by following a general "indirect" procedure in which the shear rate was taken as the independent variable instead of the lateral position (in this case we cannot say that the solution is fully analytical). That procedure was adopted by Walters [9] who derived solutions for a general linear equation of state in a number of flow configurations, and much later Schaftingen and Crochet [10] followed the same steps to give an implicit semi-analytical solution for pipe flow of the Johnson - Segalman fluid. For the Giesekus model, in the absence of solvent stress contribution, analytical solutions for channel and pipe flows were first given by Yoo and Choi [11]. The final expressions were not totally explicit, as a non-linear equation had to be solved for the pressure-gradient, and so some form of iteration was still required. Later, Schleiniger and Weinacht [12] derived identical expressions for the "classical solution" of Poiseuille flow of the Giesekus fluid, but have also analyzed "weak solutions" and gave semi-analytical expressions for the case with solvent contribution, in which an implicit procedure along the lines of that of Oldroyd and Walters is required. For the Phan-Thien/Tanner model (with parameter $\xi=0$ ), we have derived analytical expressions for both the linear and exponential forms of the model [13] and have also considered the related thermal problem including viscous dissipation [14]. Generally, it is easier to obtain a solution when the axial pressure gradient is known. However, we are more interested in the situation in which the average velocity (or flow rate) is known and the pressure-gradient unknown, as this corresponds to the situation most often found in practice. It is this situation, for the FENE-P fluid, which is analyzed here.

\section{Analysis}

The FENE-P model is based on the kinetic theory for finitely extensible dumbbells and, when the Peterlin approximation for the average spring force is introduced, it leads to a differential constitutive equation which was given in the original paper (Bird et al. [5]) in terms of the extra stress tensor $\boldsymbol{\tau}$. Following Chilcott and Rallison [15], we prefer to work here with the model expressed in terms of a configuration tensor $\mathbf{A}$ defined by $\mathbf{A} \equiv 3\langle\mathbf{Q Q}\rangle / Q_{e}{ }^{2}$ where $\mathbf{Q}$ is the end-to-end vector connecting the dumbbell beads, $\langle\cdot\rangle$ indicates an ensemble average over the configuration space, and $Q_{e}$ is an equilibrium length. The connector force of the spring in the original FENE model follows the expression proposed by Warner:

$\mathbf{F}^{c}=\frac{H}{1-(\mathbf{Q} \cdot \mathbf{Q}) / Q_{0}{ }^{2}} \mathbf{Q}$,

where $H$ is the spring constant and $Q_{0}$ the maximum possible spring length. In order to derive an evolution equation for the configuration tensor, the average $\left\langle\mathbf{Q F} \mathbf{F}^{c}\right\rangle$ is required, and it is not possible to obtain a closed-form equation unless some form of approximation is introduced. In the FENE-P model ( $\mathrm{P}$ for Peterlin), Eq. (1) for the spring force is approximated by:

$\mathbf{F}^{c} \approx \frac{H}{1-\langle\mathbf{Q} \cdot \mathbf{Q}\rangle / Q_{0}{ }^{2}} \mathbf{Q} \equiv f H \mathbf{Q}$,

following an idea suggested by Peterlin (see Bird et al. [5]). By using the definition of $\mathbf{A}$ given above, we see that the dimensionless function $f$ defined by the last term in Eq. (2) depends on the trace of $\mathbf{A}$ and can be written as:

$f \equiv f(\operatorname{tr} \mathbf{A})=\frac{L^{2}}{L^{2}-\operatorname{tr} \mathbf{A}}$, 
where $L^{2} \equiv 3 Q_{0}{ }^{2} / Q_{e}{ }^{2}$ is called the extensibility parameter of the model. It represents the square of the ratio between the maximum and the equilibrium lengths of the spring, and it is related to " $b$ " used in the original Ref. [5] by $L^{2}=b+3$ (Note: $b \equiv H Q_{0}{ }^{2} / k T ; k$ is the Boltzmann constant and $T$ the absolute temperature).

At this point it is possible, after ensemble averaging the equations of motion for the dumbbells, to derive the evolution equation for the configuration tensor of the FENE-P model as ([5], [16]):

$\stackrel{\nabla}{\mathbf{A}}=-\frac{1}{\lambda}(f \mathbf{A}-a \mathbf{I})$

which needs to be solved together with Kramers' form for the relation linking A to the polymer stress,

$\boldsymbol{\tau}=\frac{\eta_{p}}{\lambda}(f \mathbf{A}-a \mathbf{I})$

In these equations the constant model parameters are the polymer viscosity $\eta_{p}$, the relaxation time $\lambda$, and the extensibility parameter $L^{2}$. The additional parameter $a$ is not an independent parameter; it is a short notation for $a \equiv 1 /\left(1-3 / L^{2}\right)$ which arises in the derivation. It is related to physical properties by $a=1+3 k T / H Q_{0}{ }^{2}$ and to the original $b$ parameter [5] by $a=(b+3) / b$. Some times a more simplified version of FENE-P is utilized, in which $a=1$ on the assumption that $L^{2}$ is large. However, there is a certain tendency seen in the recent literature to adopt low values for $L^{2}$ (e.g. [16]), and in this case it is not adequate to consider $a=1$, so we leave it in the model equations and take account of it in the analysis.

The symbol $\nabla$ in Eq. (4) is used to denote Oldroyd's upper convected derivative,

$\stackrel{\nabla}{\mathbf{A}}=\frac{\mathrm{DA}}{\mathrm{D} t}-\mathbf{A} \cdot \nabla \mathbf{u}-\nabla \mathbf{u}^{T} \cdot \mathbf{A}$,

where $\mathbf{u}$ is the velocity vector, the material derivative is $\mathrm{D} / \mathrm{D} t \equiv \partial / \partial t+\mathbf{u} \cdot \boldsymbol{\nabla}$, and $\boldsymbol{\nabla} \mathbf{u}^{T}$ is the transpose of the velocity gradient. By combining Eqs. (4) and (5), we have:

$\stackrel{\nabla}{\mathbf{A}}=-\frac{\boldsymbol{\tau}}{\eta_{p}}$

This constitutive equation is to be solved in conjunction with the continuity and momentum equations, respectively:

$\boldsymbol{\nabla} \cdot \mathbf{u}=0 \quad$ and $\quad \varrho \frac{\mathrm{Du}}{\mathrm{D} t}=-\boldsymbol{\nabla} p+\boldsymbol{\nabla} \cdot \boldsymbol{\tau}$,

where incompressible flow is assumed and $p$ is the pressure. In general, the operator $\nabla$ satisfies:

$(f \stackrel{\nabla}{\mathbf{A}})=f(\stackrel{\nabla}{\mathbf{A}})+\mathbf{A} \frac{\mathrm{D} f}{\mathrm{D} t}$

for any function $f$. But, for the situation of fully-developed, steady and rectilinear flow, in which the only nonzero velocity component is $u(v=w=0)$, which is aligned with the flow direction $x$, we have:

$\frac{\mathrm{D} f}{\mathrm{D} t}=\frac{\partial f}{\partial t}+u \frac{\partial f}{\partial x}+v \frac{\partial f}{\partial y}+w \frac{\partial f}{\partial x}=u \frac{\partial f}{\partial x}=0$, 
and so, from Eq. (9),

$(f \stackrel{\nabla}{\mathbf{A}})=\stackrel{\nabla}{\mathbf{A}}$

It is this result which allows an analytical solution to be obtained.

If we apply the upper convected operator $\nabla$ to Eq. (5), we obtain

$\stackrel{\nabla}{\boldsymbol{\tau}}=\frac{\eta_{p}}{\lambda}((f \stackrel{\nabla}{\mathbf{A}})-a \stackrel{\nabla}{\mathbf{I}})=\frac{\eta_{p}}{\lambda}(f \stackrel{\nabla}{\mathbf{A}}+2 a \mathbf{D})$

where we used the result $\stackrel{\nabla}{I}=-2 \mathrm{D}$, with the rate-of-strain tensor denoted by $\mathbf{D}=\frac{1}{2}\left(\nabla \mathbf{u}+\nabla \mathbf{u}^{T}\right)$. The latter equation is made explicit on $\stackrel{\nabla}{\mathbf{A}}$ and equated to (7), to obtain a final expression for the FENE-P model in terms of the extra stress:

$\lambda \stackrel{\nabla}{\mathbf{\tau}}+f \boldsymbol{\tau}=2 a \eta_{p} \mathbf{D}$

The function $f$ should now be expressed in terms of the main dependent variable $\boldsymbol{\tau}$, and this is accomplished by taking the trace of Eq. (5) to get:

$\operatorname{tr} \mathbf{A}=\frac{3 a+\frac{\lambda}{\eta_{p}} \operatorname{tr} \boldsymbol{\tau}}{f}$

which is then introduced into the definition of $f$ (Eq. 3) to yield:

$f=1+\frac{3 a+\frac{\lambda}{\eta_{p}} \operatorname{tr} \boldsymbol{\tau}}{L^{2}}$.

For the problem under consideration the constitutive equation (11) reduces to the set:

$f \tau_{x x}=2 \lambda \tau_{x y} \frac{\mathrm{d} u}{\mathrm{~d} y}$,

$f \tau_{y y}=0$,

$f \tau_{x y}=a \eta_{p} \frac{\mathrm{d} u}{\mathrm{~d} y}+\lambda \tau_{y y} \frac{\mathrm{d} u}{\mathrm{~d} y}$,

which is valid for both the plane and axisymmetric cases (with the radial coordinate $r$ substituted for the lateral coordinate $y$ ). The continuity equation is satisfied identically, and the momentum equation $(8)$ reduces to:

$\tau_{x y}=P y \quad$ and $\quad \tau_{x r}=P \frac{r}{2}$

for the plane and axisymmetric cases, respectively, with $P \equiv \mathrm{d} p / \mathrm{d} x$ denoting the applied (but unknown) pressure gradient. After dividing Eq. (13) by Eq. (15), so that the function $f$ cancels out, we obtain for the normal stress:

$\tau_{x x}=\frac{2 \lambda}{a \eta_{p}} P^{2} y^{2} \quad$ and $\quad \tau_{x x}=\frac{2 \lambda}{a \eta_{p}} P^{2} \frac{r^{2}}{4}$. 
Once $\tau_{x x}=\operatorname{tr} \tau$ is known, the function $f$ can be determined from (12), and Eq. (15) gives directly the velocity gradient, which is conveniently expressed in nondimensional form as

$$
\begin{aligned}
& \frac{\mathrm{d} \hat{u}}{\mathrm{~d} \hat{y}}=-\frac{3 X}{a} \hat{y}\left[1+\frac{3 a+18 D e^{2} X^{2} \hat{y}^{2} / a}{L^{2}}\right] \text { (slit), } \\
& \frac{\mathrm{d} \hat{u}}{\mathrm{~d} \hat{r}}=-\frac{4 X}{a} \hat{r}\left[1+\frac{3 a+32 D e^{2} X^{2} \hat{r}^{2} / a}{L^{2}}\right] \text { (tube), }
\end{aligned}
$$

after scaling $y$ (or $r$ ) with the half-slit width $H$ (or the tube radius $R$ ) and $u$ with the average velocity $U$. So in Eqs. (18), and in what follows, we have introduced the notation $\hat{u} \equiv u / U$, $\hat{y} \equiv y / H, \hat{r} \equiv r / R$, and the Deborah number, used to characterize viscoelastic effects, is defined as usually, $D e=\lambda U / H$ or $D e=\lambda U / R$. The nondimensional pressure gradient parameter $X$ is defined as

$X \equiv \frac{U_{N}}{U}, \quad$ with $\quad U_{N}=\frac{-P H^{2}}{3 \eta_{p}}$ (slit) or $\quad U_{N}=\frac{-P R^{2}}{8 \eta_{p}}$ (tube)

and it is clear that $U_{N}$ has the meaning of the average velocity for the Newtonian case. Integration of Eqs. (18) from a general lateral position ( $\hat{r}$ or $\hat{y})$ to the wall $(\hat{r}=\hat{y}=1)$, where a no-slip boundary condition is imposed, gives the velocity profiles:

$$
\begin{aligned}
& \hat{u}(\hat{y})=\frac{3}{2} X\left(1-\hat{y}^{2}\right)\left[1+9 \frac{D e^{2} X^{2}}{a^{2} L^{2}}\left(1+\hat{y}^{2}\right)\right](\text { slit }), \\
& \hat{u}(\hat{r})=2 X\left(1-\hat{r}^{2}\right)\left[1+16 \frac{D e^{2} X^{2}}{a^{2} L^{2}}\left(1+\hat{r}^{2}\right)\right](\text { tube }) .
\end{aligned}
$$

It is interesting to note that the first two terms in the brackets of Eq. (18), $\left(1+3 a / L^{2}\right) / a$, become equal to unity due to the definition of $a$, with the consequence that the velocity profiles (20) reduce to the parabolic Newtonian profile whenever $D e$ tends to zero (irrespective of $L^{2}$ ), as it should. Otherwise, the solution at $D e=0$ would still depend on the viscoelasticrelated parameter $L^{2}$, in what would be an incorrect conclusion. A further integration of the velocity profiles across the slit or tube sections, together with the definitions of the average velocity in the cross section, which in nondimensional terms are:

$1=\int_{0}^{1} \hat{u}(\hat{y}) \mathrm{d} \hat{y}$ (slit) and $1=\int_{0}^{1} 2 \hat{u}(\hat{r}) \hat{r} \mathrm{~d} \hat{r}$ (tube)

gives the following cubic equation for $X$ :

$1=X\left(1+\beta X^{2}\right)$

with

$\beta=\frac{54}{5} \frac{D e^{2}}{a^{2} L^{2}}$ (slit) $\quad$ or $\quad \beta=\frac{64}{3} \frac{D e^{2}}{a^{2} L^{2}}$ (tube).

The real solution of this cubic equation is:

$$
X=\frac{432^{1 / 6}\left(D^{2 / 3}-2^{2 / 3}\right)}{6 \beta^{1 / 2} D^{1 / 3}}
$$


with:

$D=(4+27 \beta)^{1 / 2}+3^{3 / 2} \beta^{1 / 2}$,

and where the appropriate $\beta$, from Eq. (21), should be used for the slit or the tube flow.

At this stage the pressure-gradient parameter $X$ is known Eq. (22), the velocity profile is given by Eqs. (20), and the stress components can be obtained from Eqs. (16) and (17). After being scaled with the wall shear stress for the Newtonian case, the stress components are written in nondimensional form as:

$$
\begin{aligned}
& T_{x y} \equiv \tau_{x y} /\left(3 \eta_{p} \frac{U}{H}\right)=-X \hat{y} \text { (slit) } \\
& T_{x r} \equiv \tau_{x r} /\left(4 \eta_{p} \frac{U}{R}\right)=-X \hat{r} \text { (tube) }
\end{aligned}
$$

for the shear stress components, and

$$
\begin{aligned}
& T_{x x} \equiv \tau_{x x} /\left(3 \eta_{p} \frac{U}{H}\right)=6 D e X^{2} \hat{y}^{2} / a \text { (slit) } \\
& T_{x x} \equiv \tau_{x x} /\left(4 \eta_{p} \frac{U}{R}\right)=8 D e X^{2} \hat{r}^{2} / a \text { (tube) }
\end{aligned}
$$

for the normal stress components. It is noted that the wall shear stress for the upper-convected Maxwell model, for example, is identical to that for the Newtonian fluid. In this sense, it is better to view the normalized stresses in Eqs. (23) and (24) as being scaled by a factor proportional to a constant viscosity $\eta_{p}$ multiplied by a constant typical shear rate, $U / H$ or $U / R$.

\section{Discussion and conclusions}

The physical interpretation of the results is facilitated by a few graphs showing the variation of velocity and stresses. Representative velocity profiles for the tube flow are shown in Fig. 1 for fixed extensibility parameter $L^{2}=10$ and increasing Deborah number, and in Fig. 2 for fixed $D e=2$ and increasing $L^{2}$. The range of $L^{2}(10,100$ and 1000$)$ is that usually found in works with the FENE-P or FENE-CR (constant viscosity, [15]) models (e.g., [15], [16]). It is seen that both an increase of the Deborah number or a decrease of the extensibility parameter lead to flatter velocity profiles as a consequence of enhanced shear thinning in viscosity.

When $L^{2}$ tends to infinity the function $f$ tends to unity (cf. Eq. (3)), and the constitutive equations (4) and (5) reduce to the well-known upper-convected Maxwell (UCM) model, written in terms of $\boldsymbol{\tau}$ as $\lambda \overrightarrow{\boldsymbol{\tau}}+\boldsymbol{\tau}=2 \eta_{p} \mathbf{D}$ (this is more directly seen from Eq. (11) with $f \rightarrow 1$ and $a \rightarrow 1$, as $L^{2} \rightarrow \infty$ ). The UCM model is a particular form of the Oldroyd-B family with a vanishing solvent viscosity; it represents a fluid molecule with infinite extensibility and has a very simple solution in fully developed flow: the velocity profile is parabolic as for the Newtonian fluid, and the normal stress is quadratic in the velocity gradient, $\tau_{x x}=2 \lambda \eta_{p}(d u / d y)^{2}$. This trend of $L^{2}$ on the velocity variation is clearly seen in Fig. 2 when $L^{2} \rightarrow \infty$, with the shape of the profile approaching that for the Newtonian fluid; the velocity solution Eq. (20) gives this limit readily: $L^{2} \rightarrow \infty \Rightarrow X \rightarrow 1 \mathrm{Eq}$. (21), so the term in the square brackets of Eq. (20) goes to unity, and the parabolic profile is recovered. 

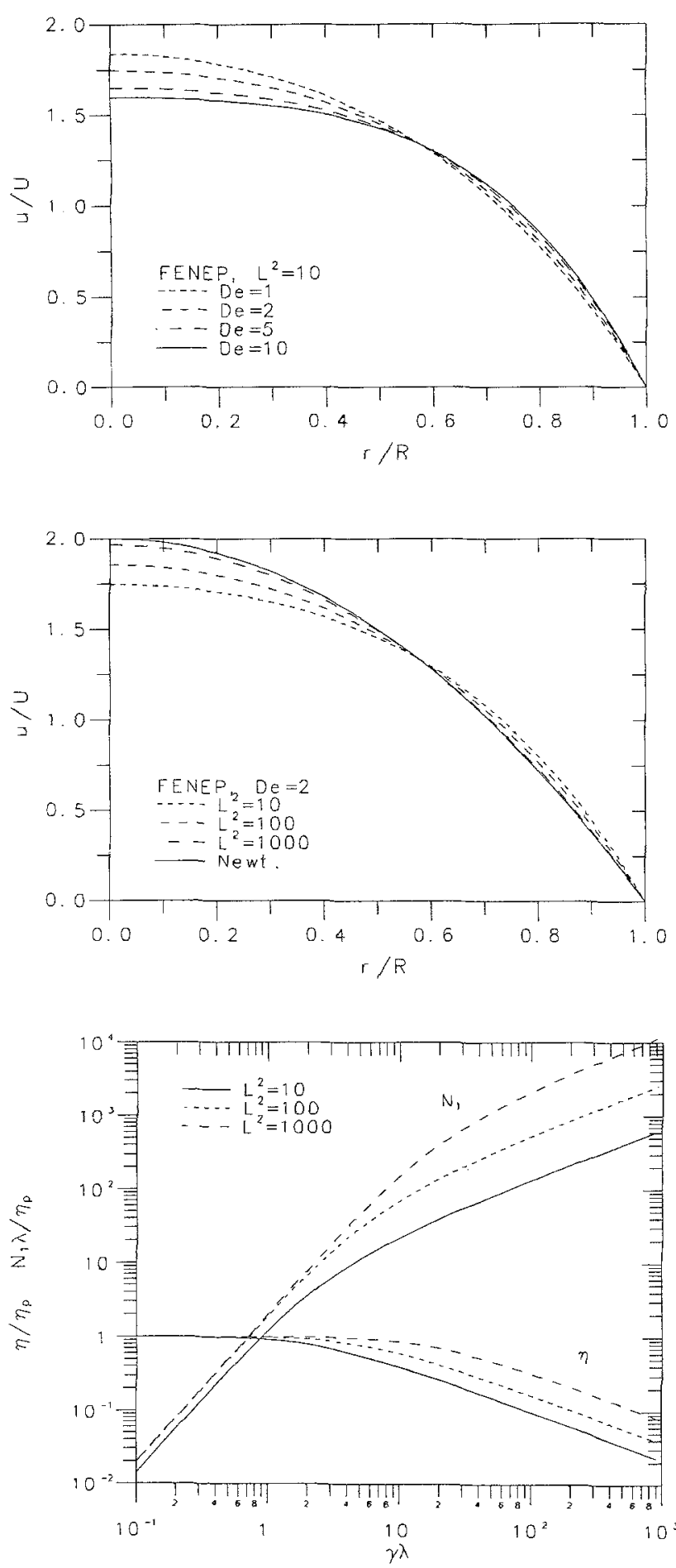

Fig. 1. Velocity profiles in tube flow for varying Deborah number (fixed $\left.I^{2}=10\right)$

Fig. 2. Velocity profiles in tube flow for varying extensibility parameter of the FENE-P model, $L^{2}$ (fixed $D e=2$ )

Fig. 3. Shear viscosity and first normal stress difference for the FENE-P fluid in simple shear flow

At this point it is worth emphasizing that although the viscosity parameter of the model $\eta_{p}$ is a constant, the viscosity function as defined by $\eta(\dot{\gamma}) \equiv \tau_{x y} / \dot{\gamma}$ in a simple shear flow (of shear $\dot{\gamma})$ is not, and tends to decrease with $\dot{\gamma}$. This is illustrated in Fig. 3 where the material properties (viscosity $\eta(\dot{\gamma})$ and first normal stress difference $N_{1}(\dot{\gamma})$, e.g. [16]) of the FENE-P fluid in simple shear flow are given as a function of the dimensionless shear rate (equal to $\lambda \dot{\gamma}$, and so 


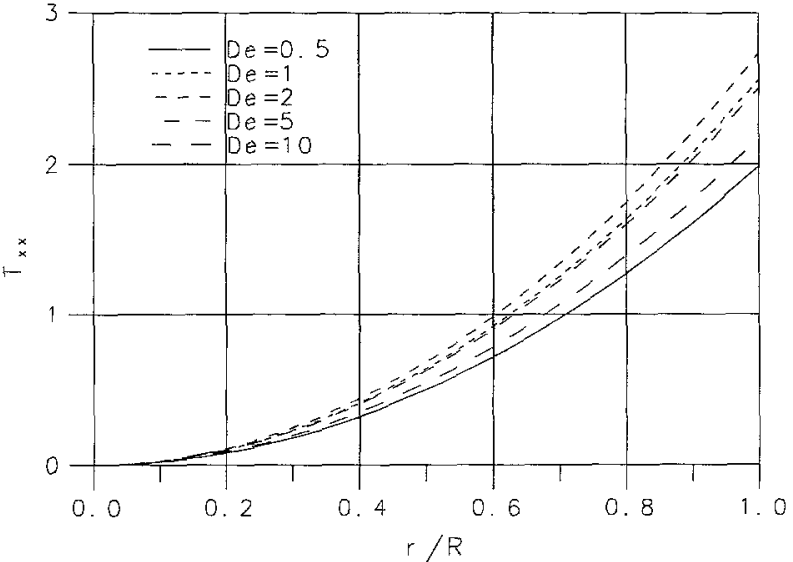

a

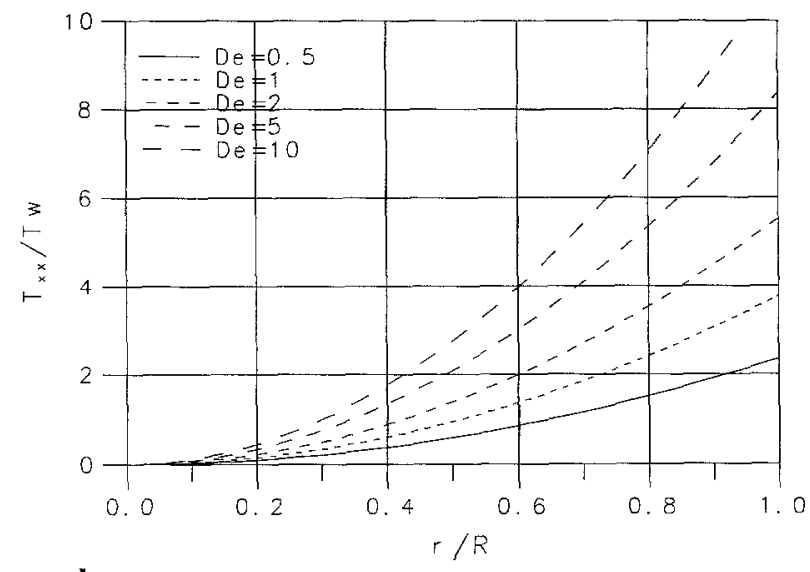

b
Fig. 4. Normal stress profiles for varying Deborah number (fixed $L^{2}=10$ ); a normalized with the Newtonian wall shear stress; b normalized with the FENE-P wall shear stress $\left(T_{W}=T_{x r}\right.$ $(r=1))$

it can be viewed as a Deborah, or more appropriately Weissenberg number). There is shear thinning in both viscosity and first normal stress coefficient $\Psi_{1}=N_{1} / \dot{\gamma}^{2}$; for large $\dot{\gamma}$, viscosity decreases as $\eta(\dot{\gamma}) \sim \dot{\gamma}^{-2 / 3}$ and normal stress increases as $N_{1} \sim \dot{\gamma}^{2 / 3}$, a well-known result [16]. For the tube and slit flows under consideration, things are not so simple because the shear rate $\dot{\gamma}$, equal to $d u / d r$ or $d u / d y$, is itself an unknown of the problem (given by Eqs. (18)) which varies over the cross section and depends in a complex way on both $D e$ and $L^{2}$.

The stress components $\left(T_{x r}\right.$ and $T_{x x}$ ) also show the expected effect of shear thinning, but the normal stress $T_{x x}$ exhibits a non-monotone behaviour with $D e$ (Fig. $4 \mathrm{a}$ ): $T_{x x}$ first increases with $D e$ (at fixed $L^{2}=10$ ), but at higher $D e$ it shows the opposite trend. This is because $T_{x x}$ is affected by elasticity (directly proportional to $D e$ ) and also by shear thinning (inversely proportional to the $4 / 3$ power of $D e$ ), c.f. Eqs. (24) and (21) at high $D e$. For this reason, it is better to plot the normal stress scaled with the wall shear stress of the FENE-P fluid, as shown in Fig. $4 \mathrm{~b}$ where profiles of $\tau_{x x} /\left(\tau_{x r}\right)_{\text {wall }}$ are given, thus removing the shear-thinning effect. A similar conclusion was found for the Phan-Thien/Tanner model [13], and for the Giesekus model as well [17], and therefore it is reasonable to admit that it represents a general feature of constitutive models exhibiting shear thinning.

For the situation depicted in Fig. 4, with $L^{2}=10$, the critical De corresponding to the maximum level of $T_{x x}$ is around 2; for higher $L^{2}$, the critical $D e$ is increased. This is illustrated 

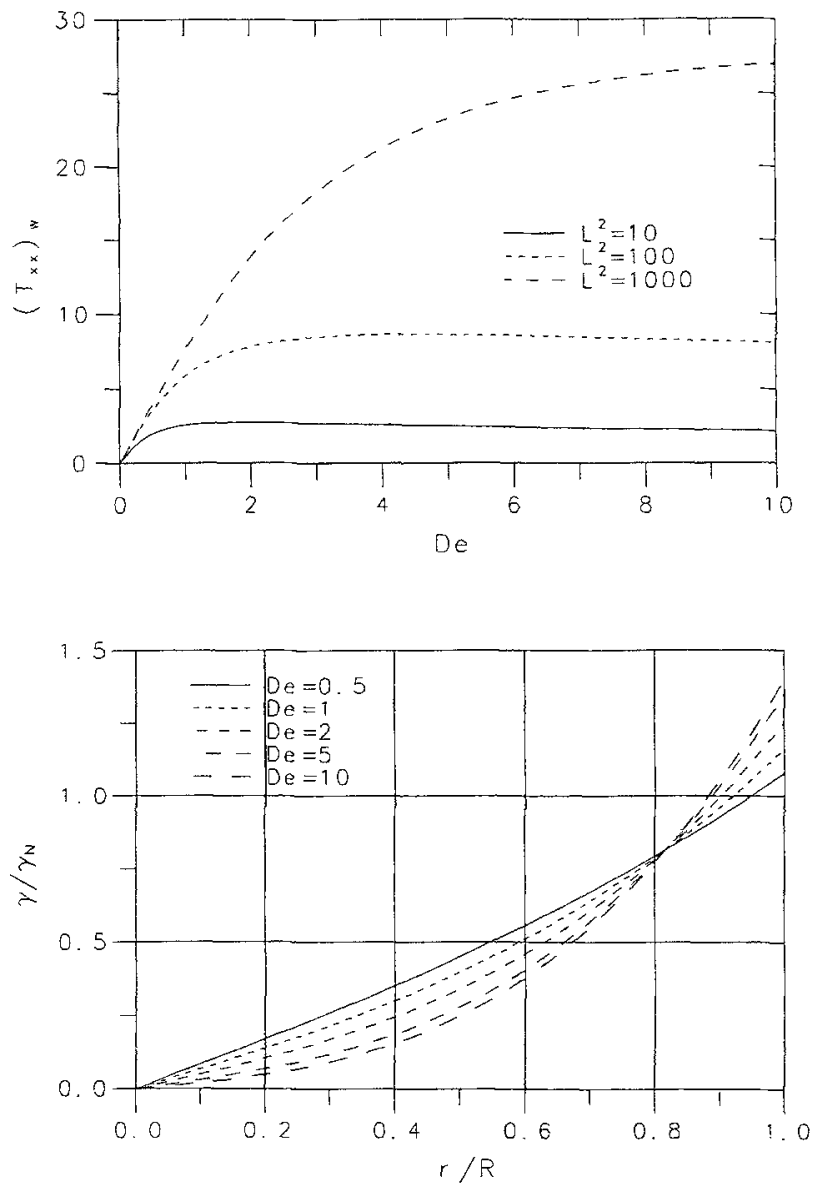

Fig. 5. Variation of the wall normal stress $\left(\left(T_{x x .}\right)_{u}\right)$ with $D e$ for various values of the extentional parameter $L^{2}$

Fig. 6. Profiles of the shear rate in tube flow for varying Deborah number (fixed $L^{2}=10$ )

in Fig. 5 which shows the normal stress at the wall $\left(\left(\tau_{x x}\right)_{w}=\tau_{x x}(\hat{r}=1)\right)$, normalized with the fixed scale $4 \eta_{p} U / R$, as a function of the overall Deborah number $D e$. It is possible to find the critical Deborah number $D e_{\mathrm{c}}$ giving a local maximum of the wall normal stress for any given $L^{2}$ by calculating the derivative of $\tau_{x x}$ with respect to $D e$ (from Eq. (24)) and equating it to zero. The final result is not amenable to an analytical expression but we have found, based on a numerical solution, that it can be conveniently expressed by the correlation:

$D e_{c}=0.507\left(L^{2}\right)^{0.476}$.

This correlation enables the calculation of the critical Deborah number to within $2 \%$ for $L^{2}$ above 10 , say from 30 up to 1000 . For lower values of $L^{2}\left(L^{2} \leq 10\right), D e_{c}$ actually increases with decreasing $L^{2}$, due to the effect of the parameter $a$ in the solution, but this range of $L^{2}$ is of little practical interest.

There is no contradiction between the above finding on the maximum of the $\tau_{x x}$ variation and the fact that the first normal stress difference is a monotonic increasing function of $\lambda \dot{\gamma}$, as seen in Fig. 3. Recall that $D e$ is a global nondimensional group characterizing the viscoelasticity of the flow, and should not be confused with a possible local Deborah number defined as $D e^{t} \equiv \lambda \dot{\gamma}$, with $\dot{\gamma}=d u / d r$. To show how complex the situation would be, we present in Fig. 6 profiles of normalized shear rate (from Eq. (18) for tube flow, divided by the factor 4 ) at $L^{2}=10$ and for increasing values of $D e$. It can be seen that there is a change of the trend on the variation of $\dot{\gamma}$ with $D e$ between the core of the flow and the near wall region. This feature 

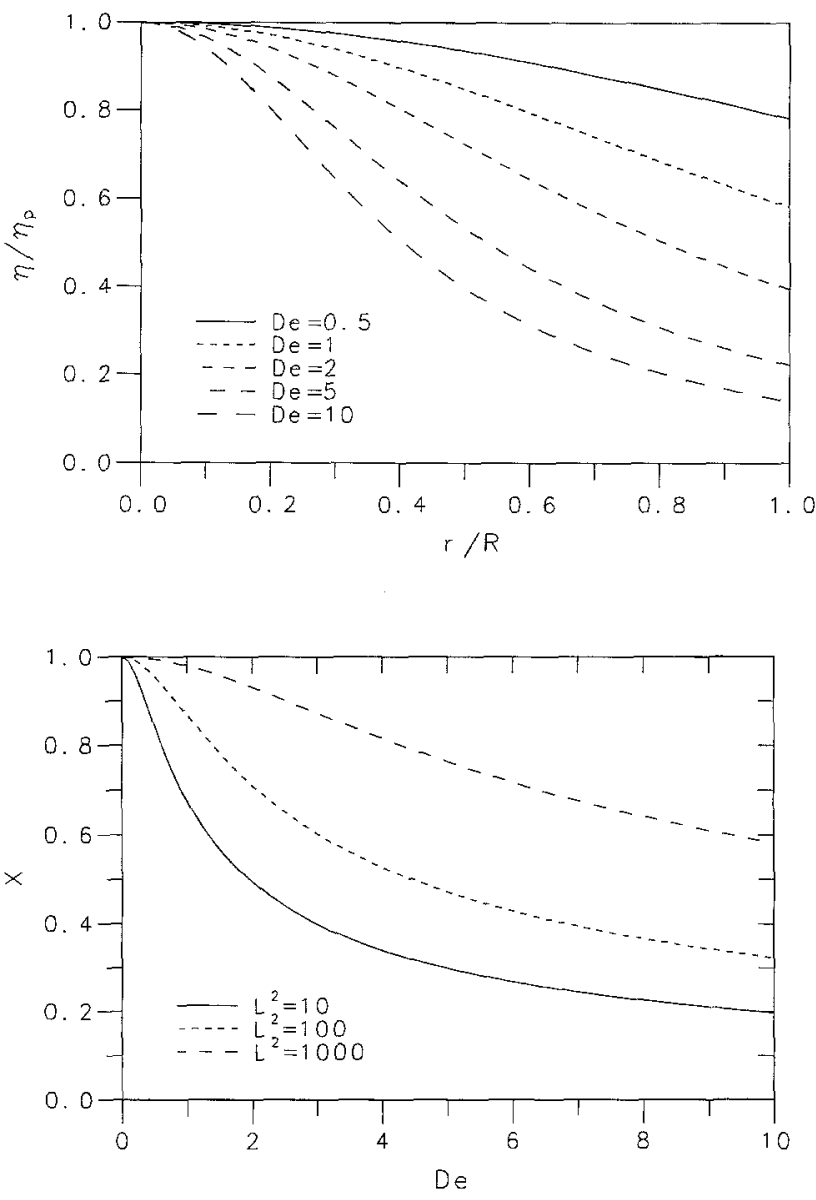

Fig. 7. Profiles of the shear viscosity function in tube flow for varying Deborah number (fixed $L^{2}=10$ )

Fig. 8. Variation of the pressure-gradient parameter $X=U_{N} / U$ with the Deborah number, at various $L^{2}$ (tube flow)

will certainly provoke the non-monotone behavior of the normal stress seen in Fig. 4 a. The viscosity function $\eta(\dot{\gamma})$, on the other hand, shows the expected shear thinning behaviour, with the $\eta(\dot{\gamma})$ versus $r$ profile decreasing all over the tube cross-section when De is raised (Fig. 7).

In what regards an equivalent to the well-known Hagen-Poiseuille result for the flow rate $(Q)$ versus the pressure-drop $(\nabla p)$, which is $Q_{N}=\pi R^{4} \nabla p / 8 \mu \ell$ for Newtonian fluids (viscosity $\mu$ ) in a tube with length $\ell$, we obtain for any constitutive model:

$\frac{Q}{Q_{N}}=\frac{1}{X}$

where, for the FENE-P fluid in particular, $X$ is given by Eq. (22). As a consequence, the flow rate increases with the Deborah number and decreases with the extensibility parameter of the FENE-P model (see Fig. 8). At high elasticity, the asymptotic behavior is $Q / Q_{N} \sim(D e / L)^{2 / 3}$.

In conclusion, an analytical solution for the flow of a FENE-P fluid in ducts of circular or planar cross-section was derived and is given in terms of velocity profiles Eqs. (20), stress profiles Eqs. (23) and (24) and shear rate Eqs. (18). The most important effect was found to be that due to shear thinning, inducing flatter velocity profiles and lower shear stresses. However, for the normal stress there is a competing influence of elasticity and shear thinning. For values of $D e$ below a critical level (given by Eq. (25)), elasticity increases the normal stresses in the cross section; for higher values of $D e$, shear thinning reverses this trend, and the normal stresses tend to decrease with $D e$. 


\section{References}

[1] Hayat, T., Asghar, S., Siddiqui, A. M.: Periodic unsteady flows of a non-Newtonian Fluid. Acta Mech. 131, $169-175$ (1998).

[2] Hayat, T., Asghar, S., Siddiqui, A. M.: On the moment of a plane disk in a non-Newtonian Fluid Acta Mech. 136, 125-131 (1999).

[3] Hayat, T., Siddiqui, A. M., Asghar, S.: Some simple flows of an Oldroyd-B fluid. Int. J. Engng Sci. 39, 135-147 (2001).

[4] Phan-Thien, N., Tanner, R. I.: A new constitutive equation derived from network theory. J, NonNewtonian Fluid Mech 2, 353-365 (1977).

[5] Bird, R. B., Dotson, P. J., Johnson, N. L.: Polymer solution rheology based on a finitely extensible bead-spring chain model. J. Non-Newtonian Fluid Mech. 7, 213-235 (1980).

[6] Giesekus, H.: A simple constitutive equation for polymer fluids based on the concept of the deformation dependent tensorial mobility. J. Non-Newtonian Fluid Mech. 11, 69-109 (1982).

[7] Rivlin, R. S.: Poiseuilie flow in a circular tube. Proc. Camb. Phil. Soc. 45, 88-91 (1949).

[8] Oldroyd, J. G.: Non-Newtonian effects in steady motion of some idealized elastico-viscous liquids. Proc. Royal Soc. A 245, 278-297 (1958).

[9] Walters, K.: Non-Newtonian effects in some elastico-viscous liquids whose behaviour at small rates of shear is characterized by a general linear equation of state. Q. J. Mech. Appl. Math. 15, 1, 63-76 (1962).

[10] Van Schaftingen, J. J., Crochet, M. J.: Analytical and numerical solution of the Poiseuille flow of a Johnson - Segalman fluid. J. Non-Newtonian Fluid Mech. 18, 335-351 (1985).

[11] Yoo, J. Y., Choi, H. C.: On the steady simple shear flows of the one-mode Giesekus fluid. Rheol. Acta 28, 13-24 (1989).

[12] Schleiniger, G., Weinacht, R. J.: Steady Poiseuille flows for a Giesekus fluid. J. Non-Newtonian Fluid Mech. 40, 79-102 (1991).

[13] Oliveira, P. J., Pinho, F. T.: Analytical solution for fully developed channel and pipe flow of PhanThien - Tanner fluids. J. Fluid Mech. 387, 271-280 (1999).

[14] Pinho, F. T., Oliveira, P. J.: Analysis of forced convection in pipes and channels with the simplified Phan-Thien -- Tanner fluid. Int. J. Heat Mass Transf. 43, 2273-2287 (2000).

[15] Chilcott, M. D., Rallison, J. M.: Creeping flow of dilute polymer solutions past cylinders and spheres. J. Non-Newtonian Fluid Mech. 29, 381-432 (1988).

[16] Purnode, B., Crochet, M. J.: Polymer solution characterization with the FENE-P model. J. NonNewtonian Fluid Mech. 77, 1-20 (1998).

[17] Oliveira, P. J., Pinho, F. T.: Some observations from analytical solutions of viscoelastic fluid motion in straight ducts. In: Proc. XIIIth International Congress on Rheology (Binding, D. M. et al., eds.) pp. 374-376. Brit. Soc. Rheo., Vol. 2 (2000).

Author's address: P. J. Oliveira, Departamento de Engenharia Electromecânica, Universidade da Beira Interior, 6201-001 Covilhã, Portugal (E-mail: pjpo@ubi.pt) 